\title{
Las teorías sociales del humor y el estudio de la risa en la Biblia Hebrea: la interjección הֶָָ y el humor humillante*
}

\author{
Sel·lam El Ammari Alonso** \\ Universidad Complutense de Madrid \\ ORCID ID: https://orcid.org/0000-0003-0234-6681
}

La interjección הָָ aparece nueve veces en la BH. Es una vocalización que expresa la alegría por la desgracia ajena y que busca escarnecer a un objetivo, ya sea un individuo o un colectivo pertenecientes al propio grupo social del emisor o no. En consonancia con las teorías sociales del humor, que se centran fundamentalmente en la función hostil de la risa, se observa que הֶָָ refleja onomatopéyicamente un tipo particular de risa que puede ser socialmente «abrasiva» o «lubricante». Se enumeran aquí las apariciones de esta interjección en el texto bíblico, analizándola a la luz de los estudios sociológicos sobre el humor humillante y ofreciendo algunas conclusiones.

Palabras Clave: Humor agresivo; ridículo; burla; teorías de la superioridad.

The Social Theories of Humour and the Study of Laughter in the Hebrew Bible: The Interjection הֶָח AND the Disparagement Humor.- The interjection הֶָח appears nine times in the Hebrew Bible. It is a vocalization which expresses happiness for the misfortunes of an in-group/out-group individual or collective. In accordance with the social theories of humor, that study the hostile function of laughter, it can be said that הָָ expresses onomatopoeically a specific kind of laughter that can be either socially «abrasive» or «lubricant». This article examines all the instances of הָָ in the HB and considers this interjection in line with the sociological studies of the disparagement humor. Finally, some conclusions are presented.

KeYwords: Aggressive Humor; Ridicule; Mock; Superiority Theory.

\footnotetext{
* La investigación en este trabajo ha sido realizada en el marco del contrato como Personal Investigador Predoctoral en formación (FPI) del Ministerio de Economía, Industria y Competitividad (ref. BES-2017-080622), adscrito al Proyecto de I+D: «Edición Electrónica Políglota de los libros de los Reyes: Göttingen Edition and the Hebrew Bible a Critical Edition» (ref. FFI2016-75064-P), financiado por el Fondo Social Europeo.

**selammar@ucm.es

Copyright: (C) 2021 CSIC. Este es un artículo de acceso abierto distribuido bajo los términos de la licencia de uso y distribución Creative Commons Reconocimiento $4.0 \mathrm{In}$ ternacional (CC BY 4.0).
} 


\section{INTRODUCCIÓN}

Pese a que la risa es una de las respuestas más comunes al juego, lo cómico y el humor, es un fenómeno que se puede dar independientemente de ellos (vid. apdos. 2 y 3 ). Es cierto que la indagación de la concurrencia de la risa en la Biblia ${ }^{1}$ puede arrojar algo de luz al asunto del humor y lo cómico en ella: observando de qué (se) ríe el «hombre bíblico» se llegaría, obviamente, a qué cosas le resultaban risibles. Pero, ¿por tanto, también «graciosas», «cómicas», «humorísticas» ${ }^{2}$ ? Independientemente de esto, se puede estudiar la aparición de la risa en el texto bíblico atendiendo al contexto lingüístico y al literario, a su simple mención o a su expresa representación escritural, a los motivos a que obedece, o a cuál es su naturaleza. Para ello, es indispensable partir de un determinado enfoque del fenómeno de la risa y dirigir la investigación en dicho sentido, que no tiene por qué ver forzosa ni totalmente con lo cómico o humorístico.

Por ejemplo, si se parte de lo que las llamadas «teorías sociales» del humor proponen acerca de la risa, se atenderá a su papel en las interacciones

${ }^{1}$ Biblia, en este trabajo, siempre será Biblia Hebrea, y Biblia Hebrea, a efecto de citas y alusiones textuales, equivaldrá siempre a Texto Masorético según la edición de Leningradensis B19a reflejada en la BHS: Biblia Hebraica Stuttgartensia, ed. Rudolf KitTel (Stuttgart, 1997). Todas las traducciones, salvo indicación contraria, son obra del autor.

2 Véase la discusión de los términos 'gracia' (español) y 'das Komische' (alemán) como correspondientes al inglés de 'humor/humour' en Belén Santana López, Wie wird das Komische übersetzt (Berlin: Frank and Timme, 2006). En resumen, el término hegemónico en la literatura científica actual es el inglés de 'humor/humour', cuyos equivalentes en las otras dos lenguas serían los indicados, no el esperado 'humor'. Se comparte la sorpresa ante el término que la autora considera clave en español con Christian F. Hempelmann, «Key Terms in the Field of Humor», en The Routledge Handbook of Language and Humor, ed. Salvatore AtTARDo (New York: Routledge, 2017) págs. 34-48: 39-41. Para el idioma alemán no hay duda de que el término 'das Komische' es el genérico para 'humor', y que no significaría 'lo cómico', pese a que los especialistas alemanes, cuando escriben en inglés, emplean 'the comic' como "falso amigo». En castellano, y sobre todo en lenguaje coloquial, «el humor se ha convertido en un hiperónimo que se asocia inevitablemente con todo lo que de una manera $\mathrm{u}$ otra nos hace reír»; $c f$. Belén SANTANA LóPEZ, «De mariposas, telégrafos, caprichos y espejos: la traducción del humor», en Actas del II Coloquio Internacional «Escrituras de la traducción hispánica», eds. Albert FreiXa y Juan Gabriel López Guix (Barcelona: Universitat Autònoma de Barcelona, 2011) págs. 59-77: 60. 
humanas ${ }^{3}$. Las cuestiones del material risible (su construcción, desarrollo y naturaleza) quedarían en un segundo plano, pues lo que más interesaría sería «cómo», «quién»y «contra quién/qué» (se) ríe uno, no tanto «de qué» (aunque este aspecto, obviamente, resulta ineludible y es siempre aludido de alguna manera $)^{4}$.

En este artículo, en primer lugar, se intentará deslindar los fenómenos de la risa y del humor (apdo. 2), y se ofrecerá una caracterización de la primera desde un punto de vista biológico y social, centrado en el «cómo se da», «quién/contra quién» la emite y «qué consecuencias» tiene, y no filosófico, con su preocupación por «cómo y por qué funciona» (apdo. 3). Tras una breve introducción a las familias teóricas tradicionales, entre las que figura la llamada «social», se entresacará de ella la preocupación sociológica por el papel y las consecuencias del humor en las relaciones sociales, (apdo. 4). Puede parecer contradictorio con la tesis de este artículo (explicitada en el apdo. 2, a saber: que risa y humor son independientes) decidir abordar el asunto de la «risa sociológica» desde el estudio del humor en las interacciones humanas, pero es justamente el carácter comunicativo de ambos fenómenos lo que permite y promueve dicho movimiento, como se explicará. A continuación, se atenderá a la risa en la BH (apdo. 5). Se enumerarán los verbos y términos relacionados con ella, así como con la burla, la mofa y el menosprecio, pero se centrará el interés y el análisis en la vocalización de la risa mediante la interjección הָָ. Como se intentará mostrar, algunos de los resultados de los estudios acerca del humor humillante en las relaciones sociales pueden ayudar a analizar el significado de esta risa concreta y, por extensión, de la risa agresiva tan típica de algunos pasajes bíblicos. Finalmente, se expondrán algunas conclusiones al respecto (apdo. 6).

${ }^{3}$ Muchas son las denominaciones que ha recibido esta familia teórica (por ejemplo, «de la superioridad», «de la hostilidad»), llamada principalmente «social» no por las disciplinas involucradas, sino por el papel (anti)social o (anti)socializador que adjudica tanto al humor como a la risa. Vid. infra apdo. 4.

${ }^{4}$ El autor de este artículo ultima su tesis sobre la comicidad en la BH, que, al contrario de lo que aquí se hará, deja los elementos referenciales e intencionales de la risa y lo cómico en segundo plano y se centra en los aspectos retóricos o de construcción. 
2. RISA Y HUMOR

La entrada que la Encyclopaedia Judaica dedica al «humor» es un buen ejemplo de la instintiva o automática identificación humor-risa. En ella, el apartado correspondiente a la Biblia y el Talmud, sin ningún preámbulo, comienza así: «[t]he Bible mentions «laughter» 50 times» ${ }^{5}$, dando por hecho y zanjado que el humor, si por algo se caracteriza, es por la risa, y que si hay que hablar de humor en el texto bíblico, hay que empezar por ella. En realidad, lo que aparece 50 veces es la raíz hebrea שחק (o el alófono vid. infra apdo. 5). Lo que la entrada dice a continuación sobre sus valores de traducción en inglés contradice de alguna manera esta primera afirmación.

Más adelante, se expresa otra asociación muy común, sobre todo en los estudios bíblicos, la de humor-ironía: «[i]n addition to the relative frequency of laughter, many examples of humor appear in the Bible. Irony is evident [in the question people asked Moses after he took them out of Egypt]» ${ }^{6}$. Con ello, además de remachar la primera identificación, se añade esta segunda (y «evidente» en pasajes como el de Ex 14,11, según se glosa). Poco más se añade en lo tocante a la $\mathrm{BH}$, aparte de señalar que, en inglés, la raíz שחקאצחק se traduce mediante términos como «play, enjoy, insult, mock, fondle, rejoice, scoff, and laugh» ${ }^{7}$. Es decir: el hebreo bíblico pluriemplea la raíz correspondiente, pues con ella no solo alude a la risa como exteriorización física y vocal de un cierto sentimiento o estado mental, sino que incluso puede aludir a la interacción física entre individuos (acariciar) o a la vocalización verbalizada (insultar). De modo inverso y complementario, se da el caso de que el hebreo bíblico utiliza varias raíces para referirse a la risa burlona y zaheridora, y diferentes expresiones que representan su sonido o la gestualidad que la acompaña (como se verá en el apdo. 5) ${ }^{8}$.

5 Avner Ziv, «Humor», en Encyclopedia Judaica, eds. Fred Skolnik y Michael Berenbaum, vol. 9 (2a ed. Detroit: Macmillan Reference USA, 2007) págs. 590-599: 590.

${ }^{6}$ ZIV, «Humor», pág. 590; las cursivas son mías. Para la ironía en la BH, cf. Edwin M. Good, Irony in the Old Testament (Sheffield: Almond Press, $1981^{2}$ ) y Carolyn J. SHARP, Irony and Meaning in the Hebrew Bible, (Bloomington-Indianapolis: Indiana University Press, 2009).

7 Ziv, «Humor», pág. 590.

8 También The Anchor Bible Dictionary hermana humor y risa en el apartado correspondiente al Antiguo Testamento, cuyo primer subapartado se titula, precisamente, 
Parece obligatorio tratar el fenómeno de la risa cuando se quiere analizar la concurrencia del humor y lo cómico en una obra o corpus, y viceversa. Pero no todo humor es cómico, ni todo lo cómico es comedia y no toda comedia provoca risa, si es que es ese su cometido. Es más, se suele rechazar la risa como un criterio definidor del humor ${ }^{9}$. Se puede aventurar que lo contrario también es cierto: el humor no es definitivo ni definitorio en la risa, como propone Provine: «[h]umor, itself a fascinating topic [...], is a recently evolved cognitive and linguistic stimulus of some but not most human laughter» ${ }^{10}$. Otros, sin embargo, no dudan de su íntima relación en términos evolutivos ${ }^{11}$.

Para algunos, la risa sería la expresión externa de una emoción como es la «diversión» (amusement) ${ }^{12}$; otros niegan precisamente que nuestra diversión ante el humor («amusement at humor») sea una emoción, y la consideran más bien como un mecanismo crucial en la evolución de la racionalidad humana ${ }^{13}$. Con todo, parece claro que «there is not a oneto-one relationship between laughter and humour [...] since laughter exceeds humour and vice versa» ${ }^{14}$.

«Laughter and Humor»; $c f$. Edward L. GREENSTEIN, «Humor and Wit: Old Testament», en The Anchor Bible Dictionary, ed. David N. Freedman, vol. 3 (Garden City, NY: Doubleday, 1992) págs. 330-333: 330; para la identificación humor-ingenio, vid. nota 16, infra.

9 Cf. Salvatore AtTardo, Linguistic Theories of Humor (Berlin-New York: Mouton de Gruyter, 1994) pág. 3. Para algunas definiciones del humor desde diferentes disciplinas y perspectivas, $c f$. Dineh DAvIs, «Communication and Humor», en The Primer of Humor Research, ed. Victor Raskin (Berlin-Boston: De Gruyter, 2008) págs. 543-568: 543-548.

${ }^{10}$ Robert R. Provine, «Laughter as a Scientific Problem: An Adventure in Sidewalk Neuroscience», The Journal of Comparative Neurology 524:8 (2016) págs. 1532-1539: 1536; las cursivas son mías.

11 Cf. Amadeu Viana, «Humour and Laughter as Vestiges of Evolution», The European Journal of Humour Research 5:1 (2017) págs. 1-18: 1.

12 John C. Simon, «Essential Attributes of a Comprehensive Theory of Laughter», The European Journal of Humour Research 8:1 (2020) págs. 45-54: 45.

13 John Morreall, «Humor and Emotion», American Philosophical Quarterly 20:3 (1983) págs. 297-304; una opinión que matiza la idea de Monrreall y apuesta por el humor como una cuasi emoción puede encontrarse en Edward GREENBERG, «Humour as a ThreatCoding Mechanism», The European Journal of Humour Research 8:1 (2020) págs. 14-28.

14 VianA, «Humour and Laughter», pág. 2. 
Theories about laughter came to share research space with humor, in a somewhat fallacious manner», dice Isabel Ermida, que señala que la supuesta simetría entre ambos conceptos, esto es, que «humor is what causes laughter and laughter is what is caused by humor», implica la falsa correlación proporcional «between an intellectual and cognitive phenomenon, humor, and a complex neuro-physiological manifestation, laughter» ${ }^{15}$. Amy Carrell dice: «[f]or some, humor is its physical manifestation, laughter; for others, humor is the comic, the funny, or the ludicrous. For still others, humor is synonymous with wit or comedy. And so the terminological fog abounds» ${ }^{16}$.

Habría para algunos una explicación histórica de la indeterminación conceptual y la confusión terminológica en el estudio de todos estos fenómenos. Hasta el siglo XVIII, la palabra «humor» ${ }^{17}$ no era sinónimo de diversión (funniness) y todos aquellos interesados en estos asuntos (filósofos y teólogos, principalmente) escribían sobre la risa y, colateralmente, sobre la comedia. Risa que, por otra parte, ya fuese provocada por algo divertido o por unas cosquillas, era tratada por igual ${ }^{18}$. Tampoco se distinguiría en la Antigüedad, a decir de algunos especialistas, entre «reír con» o «reírse de»y, en general, la risa habría sido vista indefectiblemente como un fenómeno agresivo, estimándose, por tanto, negativamente ${ }^{19}$.

15 Isabel ERmidA, The Language of Comic Narratives. Humor Construction in Short Stories (Berlin-Boston: De Gruyter Mouton, 2008) pág. 6.

16 Amy CARrell, «Historical Views of Humor», en The Primer of Humor Research, ed. V. RAskin (Berlin-Boston: De Gruyter, 2008) págs. 303-332: 306. En relación con la supuesta sinonimia entre humor e ingenio que señala Carrell, The Anchor Bible Dictionary no tiene duda de ello: la entrada que dedica al «humor» es compartida con el «ingenio»; cf. Gary A. Herion, «Humor and Wit», en The Anchor Bible Dictionary, vol. 3, págs. 325-326. Hempelmann («Key Terms in the Field of Humor», pág. 36) dice: «[i]n the collocation wit and humor, wit came to cover the more aggressive, cerebral aspects, while humor covers the benevolent, crude aspects».

17 Sobre el devenir lexicográfico del término, así como para una historia del paulatino paso de su uso médico y físiológico al psicológico y social, $c f$. Daniel WickBerG, The Senses of Humor: Self and Laughter in Modern America (Ithaca, NY: Cornell University Press, 2016) págs. 13-45.

${ }^{18}$ Cf. John Morreall, «Philosophy and Religion», en The Primer of Humor Research, ed. Victor Raskin (Berlin-Boston: De Gruyter, 2008) págs. 211-242: 211.

$19 C f$. John Morreall, «Philosophy and Religion»; Amy CArrell, «Historical Views of Humor»; Rod A. MARTin y Thomas E. Ford, The Psychology of Humor: An Integrative Approach (Oxford: Elsevier Academic Press, 2018²). 
Lydia Amir critica duramente esta y otras «imprecise, stereotyped, and at times erroneous ways in which major philosophers' attitudes toward laughter are presented in manuals of humor research and other influential manuscripts on humor» ${ }^{20}$. Cree la autora que ni el pensamiento antiguo desconocía o desapercibía los diferentes tipos de risa, ni su actitud ante ella era tan sombría y adusta como a veces se da a entender en la literatura científica. No duda de que a partir de la Ilustración se dio «a positive attitude toward laughter along with a benign form of humor», pero eso no justificaría las «estereotipadas» asunciones mencionadas ${ }^{21}$.

Se podría aducir que la risa o sus detonantes en la Biblia son siempre agresivos y degradantes; «abrasivos», en términos sociales ${ }^{22}$. Pero eso solo es válido 1) para un cierto tipo de risa provocada por unas concretas circunstancias y 2) dependiendo del sujeto y del objeto de esa risa. Observando cuándo y cómo aparece la interjección הֶֶָ se podría entender mejor este tipo de risa, diferenciándola, como se hace en la propia $\mathrm{BH}$, de otros tipos.

\section{LA RISA}

La propia naturaleza multifacética de la risa ha hecho que sean muchas (y muy distintas) las disciplinas científicas por las que se ha extendido y compartimentado su estudio, desde la filología hasta la neurología, y desde la sociolingüística a la biología evolutiva ${ }^{23}$. Son muchas las aproximaciones a la risa y muchas las descripciones tentativas de lo que es y de lo que supone.

20 Lydia AmIR, «Taking the History of Philosophy on Humor and Laughter Seriously», The Israeli Journal of Humor Research 5 (2014) págs. 43-87: 43.

21 AmIR, «Taking the History of Philosophy on Humor and Laughter Seriously», págs. 44-45.

$22 C f$. William H. Martineau, «A Model of the Social Function of Humor», en The Psychology of Humor, eds. Jeffrey H. Goldstein y Paul E. McGHeE (New York: Academic, 1972) págs. 101-125. Se verá in extenso este modelo en el apdo. 4.1 infra.

23 Jürgen Trouvain y Khiet P. Truong, «Laughter», en The Routledge Handbook of Language and Humor, ed. Salvatore AtTARdo (New York: Routledge, 2017) págs. 340355: 340 . 
El neurocientífico Robert Provine, por ejemplo, propone esta definición: «is an instinctive human vocalization [1] that is stereotyped [2], contagious [3], associated with play and humor [4], and has great social and emotional potency [5]» ${ }^{24}$. Puede ser útil dicha caracterización, dependiendo del enfoque adoptado y del fin perseguido, porque permite considerar la risa como un fenómeno pre y para-lingüístico universal (1), reconocible (2) y que puede ser inducido (3); asociado con, pero no inherente al humor y al juego (4), y que aúna la esfera emocional del individuo y su contexto social (5). Además de útil, esta definición puede ser complementada.

Desde el «análisis de la conversación» (sociolingüística) se nos ofrecen las siguientes características de la risa: a) tiene un papel muy importante en la creación y mantenimiento de relaciones interpersonales, por su habilidad para crear afiliaciones, amistad e intimidad; b) «when it is the laugher of cruelty and triumph», sirve para burlarse de otros o menospreciarles; c) responde o acompaña a todo tipo de discursos y acciones: a menudo, pero no siempre, es una respuesta al humor, pero uno también ríe cuando está nervioso o, simplemente, porque/cuando otros ríen; d) la risa forma parte del repertorio comunicativo del hombre desde antes de la aparición del lenguaje articulado y los primates también tienen comportamientos que se asemejan a o suenan como la risa humana, sirviendo a propósitos similares; e) parece ser universal en forma y función «across diverse human languages and cultures»; f) parece ofrecer múltiples beneficios físicos y psicológicos; g) no se da ni azarosa ni incontrolablemente: en las interacciones humanas, se ríe de manera sistemática y secuencial, y organizada socialmente; se coordina con el discurso hablado e incluso con los actos sociales. En suma, «the careful study of human laughter reveals much about its forms, functions, and uses, and such study reveals much about human communication in all its facets» ${ }^{25}$.

${ }^{24}$ Provine, «Laughter as a Scientific Problem», pág. 1532; la numeración entre corchetes es mía. Desde Darwin, se viene considerando la risa como una de las más importantes señales comunicativas no verbales y como marcador universal de alegría; cf. Willibald Ruch, Lisa Wagner y Jennifer Hofmann, «A Lexical Approach to Laughter Classification: Natural Language Distinguishes Six (Classes of) Formal Characteristics», Current Psychology (2019) págs. 1-13: 1.

25 Phillip GlenN, Laughter in Interaction (Cambridge: Cambridge University Press, 2003) págs. 1-2. 
Es fácil establecer relaciones entre esta lista (a-g) y las características destacadas en la definición de Provine (1-5, supra). No obstante, las características resaltadas por Glenn se imbrican y solapan, así como se excluyen y repelen. Por ejemplo, a), b) y c): la risa de unos (a) puede hacer de menos a otros (b), ya sea voluntaria o involuntariamente (es decir, una risa jubilosa y compartida por un grupo puede sonar cruel para otro); de igual manera, una risa surgida espontáneamente en un individuo de un grupo y contagiada de inmediato al resto de sus miembros (c), creando cierta empatía (a), puede sonar hostil y despreciativa para los miembros de otro grupo (b), no contagiándose en absoluto (contra c). Por otra parte, resultaría cuando menos curioso que, dándose d), no se diese e $)^{26}$.

En lo que chocarían Provine y la sociolingüística es en g). Para el neurólogo, la risa no es tan voluntaria como parece y, por tanto, tampoco tan racional como gustaría a algunos: «overestimates of the voluntary control and rationality of laughter [have been] an impediment to traditional philosophical analyses that attempt to rationalize the irrational» ${ }^{27}$. Existirían, concretamente, dos tipos de risa: una espontánea y otra voluntaria (Duchenne y no-Duchenne; vid. infra 3.2.). Durante una conversación o interacción, las dos aparecen, pero predomina la involuntaria, como respuesta a la risa de otros (contagio), sin implicar que sea impredecible.

Resumiendo, se puede afirmar que «laughter is highly dependent on motivational and emotional states, and social context. We can define laughter, then, as a ritualized and largely stereotyped vocal act that serves as a communication» ${ }^{28}$. La risa es sonido y gesto, y ambos aspectos son reconocibles incluso cuando uno de ellos falta o no es perceptible por cualesquiera causas.

26 Véase al respecto Jan van Hooff «Laughter and Humour, and the 'Duo-In-Uno' of Nature and Culture», en The Nature of Culture, ed. Walter A. Kосн (Bochum: Studienverlag Brockmeyer, 1989) págs. 120-149.

27 Provine, «Laughter as a Scientific Problem», pág. 1533. Reír no es «decir ja-ja», dice en el mismo párrafo. La gente cree erróneamente que uno ríe, voluntaria y racionalmente, para premiar algo divertido o el comportamiento de otros.

28 Martin y Ford, The Psychology of Humor, pág. 175. 


\subsection{El sonido}

El sonido de la risa puede considerarse estereotipado, pese a las divergencias inter e, incluso, intrapersonales, pues cada circunstancia provoca o convoca un tipo particular de risa. Dicho sonido parece servir a dos propósitos principales: 1) comunicar y 2) influir o provocar una reacción en otros, que no tiene que ser benigna o positiva, pese al hincapié de cierta literatura ${ }^{29}$. En cada ataque o brote de risa se darían de media unas 16 notas, comenzando cada una de ellas por la emisión de una aspirante sorda, «a hissing ' $h$ ' sound not produced by vibration of the vocal cords», seguida inmediatamente de «a forcefully voiced vowel-like sound», lo que nos daría una «ja» ${ }^{30}$. Análisis de las vocalizaciones que siguen a la aspiración apuntan a que no son ni tan nítidas ni tan bien articuladas como las producidas en el discurso, sino que más bien «tend to be a central, unarticulated $s c h w a »{ }^{31}$. No obstante, como se ha apuntado, existen distintos tipos de risa, distinguibles por sonoridad, duración, timbre, armónicos, etc., que formarían todo un repertorio (también personal) de risas, a utilizar según el momento y la ocasión. Bachorowski et al. distinguen varios tipos: la «cantarina», la «gruñidora», la «ronca», etc ${ }^{32}$.

La risa, según Provine, es una onomatopeya compartida por el hombre y los primates, pese a que los primeros la exhalen («ja-ja») y los segundos la jadeen («aj-aj»), y su sonido es primordialmente el sonido del juego: «estoy jugando, no te voy a atacar» ${ }^{33}$. Según parece indicar Provine, si la risa no fuese estereotipada, si no tuviese una estructura sónica y rítmica subyacente y común, no sería reconocible como tal ${ }^{34}$.

29 Por ejemplo, Matthew Gervais y David S. Wilson, «The Evolution and Function of Laughter and Humor: A Synthetic Approach», The Quarterly Review of Biology 80 (2005) págs. 395-430.

30 Cf. Martin y Ford, The Psychology of Humor, pág. 180, y la abundante literatura científica que citan al respecto.

${ }^{31}$ Martin y Ford, The Psychology of Humor, pág. 182.

32 Jo-Anne Bachorowski, Moria Smoski y Michael J. Owren, «The Acoustic Features of Human Laughter», The Journal of the Acoustical Society of America 110:3, pt. 1 (2001) págs. 1581-1597.

33 «Laughter is primate onomatopoeia and may be the clearest example of the phylogeny of a human vocalization»; cf. Provine, «Laughter as a Scientific Problem», pág. 1535.

34 Provine, «Laughter as a Scientific Problem», pág. 1533. 
Salta a la vista que Provine deja de lado el componente visual, por gestual, de la risa. Eso es debido a sus intereses, que quedan claros a lo largo de su descripción (vid., por ejemplo, la nota 36).

La risa y el habla pueden darse a la vez, pero el discurso prevalece, dándose que la risa funcionaría como puntuación, por lo que su aparición no sería arbitraria:

... [s]peakers typically laugh before and after complete statements and questions, seldom interrupting phrase structure [...] The placement of laughter in speech is akin to punctuating written text and is termed the punctuation effect ${ }^{35}$.

Esto indica que, neurológicamente, ambas son vocalizaciones controladas por sistemas distintos, siendo dominante el discursivo, independientemente del medio ${ }^{36}$. En las nueve ocasiones en que הֶָָ aparece en la BH, siempre sigue al verbo de dicción, אמר, y siempre inicia la serie (vid. infra apdo. 5).

En una conversación ríe más quien habla que quienes escuchan, y los comentarios que provocan risa suelen ser, en su mayoría, remotamente humorísticos ${ }^{37}$, por lo que se puede decir que lo que prima en la risa cuando se interacciona es lo social, las relaciones entre los interlocutores, no tanto lo «humorístico».

\subsection{Risa y sonrisa}

Otra asociación (menos discutida y más o menos aceptada) es la de risa-sonrisa. No en vano, los mismos músculos se ponen en funcionamiento cuando se ríe y cuando se sonríe, siendo más fuertes y duraderas sus contracciones en el primer fenómeno; además, la risa empieza y

35 Provine, «Laughter as a Scientific Problem», pág. 1536; las cursivas son suyas.

36 Ofrece Provine («Laughter as a Scientific Problem», pág. 1536) sendas pruebas que ratificarían esta asunción: «Vocal laughter also punctuates the American Sign Language (ASL) of deaf individuals, a form of manual linguistic expression that, unlike speech, does not compete with laughter for the vocal tract [...]. Neither do emoticons (visual symbols of emotion such as LOL, «Laughing Out Loud» etc.) disrupt phrases in online text messages, a nonvocal linguistic medium».

37 Provine, «Laughter as a Scientific Problem», pág. 1536. 
termina con una sonrisa. La diferencia entre ambos fenómenos estaría en la intensidad emocional implicada ${ }^{38}$.

La sonrisa sería una de las maneras en que la risa lleva a cabo su función comunicativa $^{39}$. Se han identificado casi una veintena de tipos de sonrisa, aunque solo una está directamente relacionada con la diversión. Sería esta risa auténtica y no forzada, y se denomina Duchenne por el nombre de su descubridor ${ }^{40}$. Cuando se ríe se exhibe la variante Duchenne de la sonrisa, pues es esto último un indicador de la sinceridad o el fingimiento de la risa. Esto es muy importante, pues el tipo de gesto de una persona riente influye en el estado emocional de los receptores, habiéndose demostrado que la risa (y sonrisa) Duchenne se asocia implícitamente con la proximidad psicológica, mientras que la no-Duchenne se asocia con la distancia psicológica ${ }^{41}$.

\subsection{Sociabilidad de la risa}

Que la risa es potencial y altamente contagiosa explica la existencia de la claque en el teatro y las risas enlatadas en televisión. Su función es «señalar» los momentos cumbre o los punch lines ante los que habría que aplaudir y/o reír, pero Provine indica que no se tiene en cuenta que la risa provoca risa sin necesidad de un «chiste» y aventura que «humans may have a 'feature detector' for laughter, a neural circuit that responds exclusively to this vocalization and triggers the motor pattern of laughter in listeners ${ }^{42}$. La reacción a la risa con más risa es similar a la que provocan el llanto, el bostezo, las toses o el rascarse, y habla de la im-

38 Martin y Ford, The Psychology of Humor, pág. 175.

39 Jan VAN Hooff («A Comparative Approach to the Phylogeny of Laughter and Smiling», en Non-Verbal Communication, ed. Robert A. HindE [Cambridge: Cambridge University Press, 1972] págs. 209-238) cree que, para hombres y primates, filogenéticamente, risa y sonrisa difieren en origen, pero que han terminado por confluir en el hombre.

40 Fue el anatomista francés del siglo XIX G.B. Duchenne de Boulogne quien la descubrió. La misma distinción se aplica a otros gestos y a la propia risa; $c f$. VIANA, «Humour and Laughter». Los gestos Duchenne serían más o menos espontáneos y genuinos; todo lo contrario los no-Duchenne.

41 Martin y Ford, The Psychology of Humor, pág. 180.

42 Provine, «Laughter as a Scientific Problem», pág. 1537. 
portancia de las llamadas «neuronas espejo», que se activan tanto cuando se realiza una acción como cuando se observa ${ }^{43}$.

Esta contagiosidad y sociabilidad de la risa ha llevado a considerar que es siempre beneficiosa a efectos individuales y grupales, pero, evidentemente, es esta una asunción errónea que, hoy día, parece hegemónica y basada en una cierta ideología. Existe, sin duda, un lado oscuro de la risa que es análoga a la que el sociólogo Michael Billig señala en el humor:

It is easy to praise humour for bringing people together in moments of pure, creative enjoyment. But it is not those sorts of moments that constitute the social core of humour, but, instead, it is the darker, less easily admired practice of ridicule ${ }^{44}$.

Salta a la vista que lo mismo se puede decir de la risa, que también posee un carácter social y hace de ella una «vocalización social»: "[1] aughter, like speech, evolved to change the behavior of others, and it rarely occurs when we lack a real or vicarious audience» ${ }^{45}$. Lo que Billig señala en el párrafo anterior, y los estudios sobre, por ejemplo, la «gelotofobia» 46 analizan, es que la risa o el humor son agentes o herramientas de socialización, pero no inocuas o indefectiblemente beneficiosas. Los estudios llevados a cabo en personas con miedo a la risa ajena confirman que «humor and laughter are not relaxing and enjoyable social experiences» por defecto ${ }^{47}$. Lo que ocurre con los

43 Provine, «Laughter as a Scientific Problem», pág. 1537.

${ }^{44}$ Cf. Michael Billig, Laughter and Ridicule: Towards a Social Critique of Humour (London: SAGE Publ., 2005) pág. 2; las cursivas son mías.

45 Provine, «Laughter as a Scientific Problem», pág. 1536; las cursivas son mías.

$46 \mathrm{O}$ miedo a la risa ajena. Los orígenes del término y de la investigación de esta concreta psicopatología se deben a Michael Tiтze, «The Pinocchio Complex: Overcoming the Fear of Laughter», Humor \& Health Journal 5 (1996) págs. 1-11. Términos acuñados (o recalibrados) en la estela de este neologismo son los de «gelotofilia», o placer por ser objeto de risa/provocarla en otros, «katagelasticismo», o placer en ridiculizar a otros, y el de «estado agelótico»: cuando no se es capaz de apreciar el humor en una situación o lo beneficioso de la risa, lo que provoca la «cara de palo» a la que alude el «síndrome de Pinocho» del artículo de Titze citado al principio. Para los términos «gelotofilia» y «katagelasticismo», cf. Willibald Ruch y René T. Proyer, «Extending the Study of Gelotophobia: On Gelotophiles and Katagelasticists», Humor: International Journal of Humor Research 22:1-2 (2009) págs. 183-212.

47 Michael TiTzE, «Gelotophobia: The Fear of Being Laughed at», Humor: International Journal of Humor Research 22-1:2 (2009) págs. 27-48: 31. 
«gelotófobos», no obstante, es su incapacidad para percibir lo beneficioso de la risa y su propensión a considerarla siempre agresiva y siempre dirigida a ellos. Además, suelen confundir la alegría ajena con el desprecio hacia ellos: "positive emotions are not contagious for them» ${ }^{48}$.

Parece indudable que existen teorías sobre el humor y lo cómico que traslucen una evidente prevención, cuando no odio, ante la risa, y algunos se preguntan si no serán obra de gelotófobos: «[m]aybe we owe our misanthropic theories of humor [...] to gelotophobes [...] (e.g., the misogelos, or 'laughter haters') ${ }^{49}$. Incluso podría suceder que «the richness of the negative vocabulary in describing laughter actually stems from gelotophobes or aghelastos/misoghelus (i.e., laughter haters)» ${ }^{50}$. Pero tanto el hecho denunciado, como la expresión de la denuncia, dejan al descubierto los extremos de sendas acríticas asunciones respecto a la risa, que funcionan como punto de partida para emitir juicios de valor: «la risa es mala por defecto, luego cualquiera de sus atributos ha de serlo por fuerza»/«la risa es beneficiosa, luego el vocabulario negativo con que se la describe ha de ser, por fuerza, obra de personas con aversión a ella o carentes de humor».

De ordinario se señala que la risa en la $\mathrm{BH}$ no está muy bien vista, y no faltará quienes señalen que la «gelotofobia», «agelastia» o «misogelia» de sus creadores es lo que subyace en ello. Pero en el texto bíblico se distinguen muy bien los distintos tipos de risa, y cuando se critica la risa hostil y agresiva, o por hostil y agresiva, es porque el otro/los otros la utiliza/n o emite/n contra un correligionario (en Salmos, contra el propio salmista) o contra el pueblo en general. Cuando Yhwh, la congregación o los «buenos» se ríen y burlan del rebelde, del idólatra o del enemigo, la risa es oportuna, porque les está bien empleada.

48 Willibald Ruch, Jennifer Hofmann, T. Platt y René T. Proyer, «The State-of-the Art in Gelotophobia Research: A Review and Some Theoretical Extensions», Humor: International Journal of Humor Research 27:1 (2014) págs. 23-45: 37.

49 Ruch, Hofmann, Platt y Proyer, «The State-of-the Art in Gelotophobia Research», pág. 30 .

50 Ruch, Wagner y Hofmann, «A Lexical Approach to Laughter Classification», pág. 9. 
4. LAS TEORÍAS SOCIALES SOBRE EL HUMOR Y EL ESTUDIO DEL HUMOR HUMILLANTE

Las teorías que sobre el humor se han dado desde finales del siglo XIX pueden dividirse en 1) cognitivas o de lo incongruente, 2) psicoanalíticas y 3) sociales, de la superioridad o de la hostilidad ${ }^{51}$.

Salvatore Attardo ofrece una clasificación de los aspectos del humor que cada familia teórica estipula claves para su identificación e investigación $^{52}$ :

- Cognitivas: Contraste, incongruencia.

- Sociales: Hostilidad, agresión, superioridad, triunfo, escarnio, menosprecio.

- Psicoanalíticas: Descarga [release], sublimación, liberación, economía.

Cada familia teórica se ocuparía de una parcela distinta en lo relativo a la risa y lo cómico: la cognitiva, a la percepción de lo cómico; la social, a la función de la risa y el humor; y la psicoanalítica, a los efectos psíquicos y anímicos ${ }^{53}$. Las llamadas teorías sociales o de la superioridad se centran en la risa y en la actitud de desprecio, crítica u hostilidad por parte de sus emisores o sus receptores/espectadores hacia unos determi-

51 Recientes repasos de las tres familias, con bibliografía, son los de Noël CARroll, Humour: A Very Short Introduction (Oxford: Oxford University Press, 2013); Cristina Larkin-Galiñanes, «An Overview of Humor Theory», en The Routledge Handbook of Language and Humor, ed. Salvatore ATTARDO (New York: Routledge, 2017) págs. 4-16, y John Morreall, «Philosophy of Humor», en The Stanford Encyclopedia of Philosophy, ed. Edward N. ZaLTA (Fall 2020 Edition, accesible en línea en $<$ https://plato.stanford. edu/archives/fall2020/entries/humor $>$ ). Para una crítica total a todas las familias teóricas en conjunto, que no harían sino perpetuar prejuicios muy antiguos en torno al humor, cf. John Morreall, Comic Relief: A Comprehensive Philosophy of Humor (Malden, MA: Wiley-Blackwell, 2009).

${ }^{52}$ AtTardo, Linguistic Theories of Humor, pág. 47; la traducción es mía; para una clasificación muy similar, $c f$. Salvatore AtTARDo, «A Primer for the Linguistics of Humor», en The Primer of Humor Research, ed. Victor RASKIN (Berlin-Boston: De Gruyter, 2008) págs. 101-156: 103.

${ }^{53}$ Sheila Lintotт («Superiority in Humor Theory», Journal of Aesthetics and Art Criticism 74:4 [2016] págs. 347-358: 347) observa que las tres teorías o familias teóricas clásicas no son rivales, pues no se ocupan de lo mismo. 
nados objetivos. La característica principal de la risa así producida es la agresividad. La risa es contra un objetivo y es usada como arma contra algo o alguien: un individuo, un grupo, una clase social o política, etc. Sheila Lintott indica que esta familia de teorías es parcial y no define el humor per se, sino que se limita a explicar «the nature and value of some humor»y lamenta que, no obstante, «the theory is often presented as a stand-alone, comprehensive theory of humor» ${ }^{54}$. Lo más significativo de estas teorías ${ }^{55}$ es que no se preocupan tanto del cómo se construye o se consigue esa risa, como de (mayormente) criticarla y demonizarla ${ }^{56}$.

\subsection{El humor humillante}

En un seminal artículo de 1972, William H. Martineau se ocupó del papel o función social del humor como medio de comunicación, señalando su paradójica naturaleza:

we advocate that humor be viewed from the sociological perspective as a «lubricant» and an «abrasive» in social interaction -especially in such common everyday interaction that constitutes the basis of the social order and makes the routine flow of social life possible ${ }^{57}$.

El humor y la risa pueden expresar dos mensajes contradictorios y evitar cualquier tipo de confrontación, lo cual puede ser socialmente beneficioso o perjudicial. Por ejemplo, una crítica negativa «envuelta» en bromas y/o risas puede sofocar de antemano cualquier conato de discusión; pero también una invectiva encerrada en una carcajada deja al objetivo inerme y sin capacidad de respuesta, pues al fin y al cabo,

54 LintotT, «Superiority in Humor Theory», pág. 348.

55 Ciertamente, hasta Hobbes (s. XVII), no hubo sino opiniones y comentarios, casi siempre negativos, sobre la risa y, más concretamente, la risa burlona, pero no teorías acerca de ella; $c f$. Billig, Laughter and Ridicule, pág. 40.

56 Con todo, esta teoría es estrictamente una teoría sobre el humor, pues no se preocupa de la risa producida en otras circunstancias que no sean las que ella misma estipula humorísticas, por agresivas, y viceversa; $c f$. Claudio PAolucci y Fausto CARUANA, «Per un'etologia semiotica del riso di superiorit'a. Un'ipotesi pragmatista ed evoluzionista», Reti, Saperi, Linguaggi 6:2 (2019) págs. 243-260.

57 Martineau, «A Model of the Social Functions of Humor», pág. 103. 
las carcajadas señalarían la no seriedad del ataque y, en teoría, eso evitaría de entrada cualquier réplica: «[b]ecause humor communicates that its message is to be interpreted in a non-serious manner, disparagement humor can uniquely denigrate its target while stiflin challenge or criticism ${ }^{58}$. Para algunos, este tipo de humor es el origen de muchas aversiones y fobias a la risa ajena y a la risa en general (véase la literatura citada en 3.3.).

Martineau ideó un modelo para estudiar este tipo de humor basándose en las funciones que desempeña grupal e «inter-grupalmente». Es decir, fijó un marco basado en 1) el humor dentro de un grupo, 2) el humor intergrupal desde la perspectiva de sus repercusiones dentro de un grupo y 3) el papel del humor intergrupal desde el punto de vista de la interacción y las relaciones entre dos grupos ${ }^{59}$. Se puede ejemplificar «bíblicamente» este modelo de estudio, diciendo que consistiría en 1) analizar las risas y burlas que denuncia contra su persona el salmista de continuo, por ejemplo; 2) observar las repercusiones que las burlas de otros pueblos tienen en el de Israel, como se lee, por ejemplo, en la hímnica (Sal 44,14-16.79,4) o en la literatura profética, que no solo las señala, sino que amenaza con ellas (cf. Je 24,9-10.25,15-18); y 3) estudiar cómo las burlas entre naciones según se las describe en la $\mathrm{BH}$ son un toma y daca en que lo «lubricante» para unos es «abrasivo» para otros, como se ve en Jb 22,19-20, que describe una situación prototípica en que los participantes pueden ver sus papeles cambiados. En resumen, «[d]isparagement humor can facilitate smooth or conflicting relations, depending on its rewards or costs for a given identity ${ }^{60}$. Como la literatura posterior ha ido señalando, 2 y 3 se pueden aplicar a 1; es más, 1 englobaría micro cósmicamente 2 y 3 . De este modelo, Martineau derivó o extrajo una serie de teoremas que identificaban la función del

58 Mark A. Ferguson y Thomas E. Ford, «Disparagement Humor: A theoretical and Empirical Review of Psychoanalytic, Superiority, and Social Identity Theories», Humor. International Journal of Humor Research 21:3 (2008) págs. 283-312: 284. Quien emplea el humor con fines degradantes o humillantes lo hace sin miedo a la sanción, pues siempre puede aducir en su defensa aquello de, «no iba en serio». Eso lo hace en ocasiones especialmente dañino.

59 Martineau, «A Model of the Social Functions of Humor», pág. 115.

${ }^{60} C f$. Ferguson y Ford, «Disparagement Humor», pág. 299. 
humor dependiendo del escenario y los sujetos implicados y del juicio que de aquel tuviesen los participantes ${ }^{61}$.

Así, por ejemplo, en una situación intragrupal $(1$, vid. supra $)$, si el humor es a) considerado positivo, consolida el grupo; y si es b) juzgado como humillante o degradante, puede b1) controlar el comportamiento dentro del grupo, b2) consolidar el grupo, b3) introducir un conflicto o alentar uno ya presente, o b4) promover la desmoralización o la desintegración en el grupo. De entrada, b3) y b4) no estarían necesariamente relacionados, porque el conflicto dentro de un grupo no tiene por qué desintegrarlo. El ridículo (la ridiculización) no es, por fuerza, un desintegrante social:

Ridicule, as one type of disparaging humor, is often described as such a conflict weapon. One empirical question is to discover the proportion of severely abrasive humor which can be tolerated before necessary communication becomes impaired and the basis of social integration destroyed ${ }^{62}$.

Como se observa, «[a]s a basic medium of communication, humor assumes many forms and its social functions become complex under the influence of other social processes and existing social structures» ${ }^{63}$. Lo que la «risa social» señala es la existencia de un/os sujetos rientes a costa de unos determinados objetivos y presupone una serie de consecuencias en unos y otros en sus respectivas relaciones internas y en su interacción.

Se puede adaptar a la risa el modelo de Martineau, con independencia de la relación de aquella con lo humorístico, que puede incluso no existir. En los contextos que el modelo plantea, la risa sería el signo

${ }^{61} C f$. Martineau, «A Model of the Social Functions of Humor», págs. 116-123. El modelo de Martineau sigue vigente como piedra de toque de diversos acercamientos sociológicos y psicológicos al humor. $C f$., por ejemplo, Leslie M. JANES y James Olson, «Humor as an Abrasive or a Lubricant in Social Situations: Martineau Revisited», Humor 28:2 (2015) págs. 271-288.

62 Martineau, «A Model of the Social Functions of Humor», pág. 118. Todavía podría el humor ser considerado $c$ ) estimativo respecto de un $\mathrm{grupo}_{2}$, lo que consolidaría al grupo ${ }_{1}, \mathrm{o} d$ ) degradante o humillante respecto del $\mathrm{grupo}_{2}$, lo que podría bien 1) incrementar la moral del grupo $_{1}$, bien 2) introducir o fomentar la hostilidad respecto del grupo $_{2}$, bien 3) darse ambos circunstancias simultáneamente (cf. págs. 118-119).

63 Martineau, «A Model of the Social Functions of Humor», pág. 124. 
comunicativo que cada individuo y cada grupo juzgarían como estimativo/positivo o denigrante/humillante, y eso sería suficiente para la aplicación de los teoremas. La risa posee un carácter retórico que la permite comunicar significados y no es tanto un acto reflejo que sigue a un determinado estado mental/emocional ${ }^{64}$. Por eso, en ciertos contextos y bajo ciertas condiciones, la risa funciona como el humor y, se aventura, en ocasiones es todo «el humor» que aparece cuando se señala su participación en una situación. La risa, por sí sola, puede ser escarnio, burla y desdén, tal y como un comentario jocoso puede serlo. La risa bien antecede, bien acompaña, bien subsigue, bien sustituye a cualquier otro signo comunicativo, también verbal, que pueda ser considerado humorístico.

\section{LA RISA EN LA BH}

La $\mathrm{BH}$ es uno de los primeros textos occidentales en tratar y hablar de la risa, no solo en mencionarla ${ }^{65}$. Si tomamos la raíz שח como principal término para la risa, encontramos en Eclesiastés y Proverbios algunas valoraciones de ella. Por un lado, se la distingue claramente del disgusto y la aflicción, ensalzándose las cualidades meliorativas de la tristeza respecto de la alegría ${ }^{66}$ (Ec 7,3), se la asocia al baile, la fiesta y el jolgorio (Ec 3,4.10,19) y es expresión de gozo y deleite (Pr 8,30-31); por otro lado, se señala su locura, su necedad y su bulla (Ec 2,2.7,6) ${ }^{67}$, se la relaciona con el mal y el embuste (Pr 10,23.26,19) y no se deja de señalar que, como la alegría, es efímera y no evita el dolor $(\operatorname{Pr} 14,13)$.

${ }^{64}$ BILLIG, Laughter and Ridicule, pág. 189.

${ }^{65}$ Cf. Morreall, «Philosophy and Religion», pág. 212.

${ }^{66}$ Esto es algo que se aceptaría sin problemas entre las filas «gelotofílicas»; cf. Billig, Laughter and Ridicule, y su queja de la acrítica asunción actual de que la risa y el humor no solo son buenos por definición, sino que mejoran la calidad de vida y aún la salud de las personas.

${ }^{67}$ Cf. Etan Levine, «Qohelet's Fool: A Composite Portrait», en On Humour and the Comic in the Hebrew Bible, eds. Yehuda T. RADDAY y Athalya BREnNER (Bloomsbury Publishing Plc, 1990) págs. 277-294. Para la ambivalencia de la risa en Eclesiastés, cf. Athalya Brenner, «On the Semantic Field of Humour», en On Humour and the Comic in the Hebrew Bible, págs. 39-58: 40, n.3. 
Pero el campo semántico de la raíz שח va más allá de la risa como vocalización y gesto, pues incluye desde el juego y la diversión hasta la burla, pasando por el regocijo, el baile y la alegría ${ }^{68}$. Está relacionada con todo lo que tiene que ver con el esparcimiento, la fiesta, la música, el solaz, el regocijo y cierto desenfreno asociado a los placeres mundanos (también carnales). La forma צחק (צחק es alófono ${ }^{69}$ y una forma סח se da una vez en Qumrán ${ }^{70}$ ) aparece a menudo en combinación con sinónimos o antónimos y refleja gran amplitud semántica: desde el baile hasta el escarnio, de la fiesta a la mofa ${ }^{71}$.

La risa «biológica» y «sociológica» (vid. supra) pueden aparecer en el texto bíblico sin la participación de la concreta raíz שח que, como se ha visto, está pluriempleada en la $\mathrm{BH}$ y, por tanto, en muchas ocasiones, describe o alude a acciones o hechos muy alejados de la risa tal y como se la ha caracterizado en el apdo. 3. La risa se oye y se ve, y es perfectamente reconocible aun faltando la percepción de uno de sus componentes, ya el sonoro, ya el gestual. Por lo general, en el texto bíblico, se la suele mencionar, pero de vez en cuando, además, se la describe y es «mostrada». Así, Ec 7,6 dice que «como suenan las zarzas

${ }^{68} C f$. Brenner, «On the Semantic Field of Humor».

69 Bruce Rosentock, «Dionysus in Israel: The Semantics of ȘHQ in the Hebrew Bible», Jewish Studies Quarterly (JSQ) 19:4 (2012) págs. 289-310. BRENNER («On the Semantic Field of Humor», pág. 46) cree que «[e]tymologically and diachronically, available evidence points to the primariness of shq»».

${ }^{70}$ Cf. David J. ChINES, «Classical Hebrew: All 3,308 Byforms, Alphabetically», (s. l, $s . d$., accesible en $<$ https://www.academia.edu/34122190/Byforms_Alphabetically_pdf $>$ ) págs. 1-93: 56.

71 Para el uso de los derivados de שח cf. Ju 16,25 y 27; 1 Sa 18,$7 ; 2$ Sa 2,14, y 6,5 у 21 ; Je $15,17,20,7,30,19,31,4,48,26-27$ y 39 ; Ha 1,10; Za 8,5 ; Sal 2,4, 37,13, 52,8, $59,9,104,26$ y 126,$2 ;$ Jb $5,22,8,21,12,4,29,24,30,1,39,7,18$ у $22,40,20$ у 29 у 41,21 ; Pr $1,26,8,30-31,10,23,14,13,26,19,29,9$ y 31,$25 ;$ Ec $2,2,3,4,7,3$ y 6, y 10,19; La 1,7 y 3,$14 ; 1$ Cr 13,8 y 15,29, y 2 Cr 30,10. Para el uso del alófono צחק , sin incluir el nombre de Isaac, $c f$. Ge 17,17, 18,12-13 y $15,19,14,21,6$ y 9, 26,8 y 39,14 y 17; Ex 32,6; Ju 16,25, y Ez 23,32. צחק nunca se combina con otros sinónimos, salvo en Ju 16,25 (curiosamente, con שחק; cf. Charles Halton, «Samson's Last Laugh: The «Ś/ŠHQ̣» Pun in Judges 16,25-27», Journal of Biblical Literature 128:1 [2009] págs. 61-64 [accesible en <www.jstor.org/stable/2561016>) y Ez 23,32, donde es acompañado por לעג ("burlarse'). Una explicación de la ausencia de שחק en la Torah, puede verse en BrenNER, «On the Semantic Field of Humor», págs. 45-52 passim. 
en la marmita, así es la risa del necio»» ${ }^{72}$. Es grito triunfal, como en $\mathrm{Jb}$ 8,21 o 39,25 (aquí, casi relincho) ${ }^{73}$. En 1Sa 2,1 es expresión victoriosa con que una exultante Hannah se ríe de sus enemigos. Puede también la risa, por el contrario, no sonar en absoluto, como en Ge 18,12, donde Sarah ríe «por dentro» (帒).

La risa es gesto y mueca. Puede llenar la boca, como en Sal 126,2 y Jb 8,21, o ensancharla y casi desfigurarla, como en Sal 35,21, donde bocas enemigas profieren הֶָָח הֶָר burlones (vid. infra), que se suman a guiños (v.19) y rechinar de dientes (v.16) despectivos. En este sentido, la cantidad de gestos y aspavientos desdeñosos es abundante en la BH: manos que se agitan, cabezas que se menean, lenguas que se sacan, silbidos, etc ${ }^{74}$. La risa, por sonido y mueca, puede ser un gesto o señal más de escarnio y ludibrio, o el principal (que se elide y/o da por supuesto), pero ha de quedar claro que es este un tipo concreto de risa: agresiva, hostil y zaheridora, y que, sea cual sea su tipo, la risa es un signo comunicativo. 2Cr 30,10; Je 20,7; Ez 23,32; Sal 2,4 y Pr 1,26 emparejan risa y burla (raíz לעב לעב לער), por lo que puede ser legítimo asumir la risa en un pasaje -si se dan otros gestos relacionados, como los que expresan los pasajes citados en la nota 74- aun faltando la raíz שחק, cuanto más si aparece la interjección

72 Puede comprobarse empíricamente cómo es ese sonido, si alguien está interesado en saber a qué se le parecía la risa del necio al Eclesiastés, y quizá la aliteración y el juego

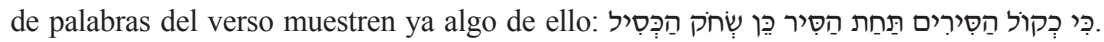

73 Cf. BDB, s. v. הֶָָח; Eliezer Ben YehudA, מלון הלשון העברית הישנה והחדשה / A Complete Dictionary of Ancient and Modern Hebrew (Tel Aviv: La’am, 1948-1959. 17 vols.) vol. 2, pág. 1022, s. v. הֶָָ. y la traducción correspondiente en Luis AlONSO SCHÖKEL, Biblia del peregrino. Edición de estudio (Estella: Verbo Divino, 1997. 3 vols.) vol. 2, pág. 951. Puede que se dude de que הֶָָ en Jb 39,25 represente o describa tan brutal carcajada, pero la aparición de la raíz שחק por triplicado en el capítulo no deja duda.

74 Véanse, por ejemplo, 2Re 19,21; Is 37,22; Je 19,8, 49,17 y 50,13; Ez 27,36; So 2,15; Sal 22,7-8, 37,12 y 109,25; Jb 16,4 y 9 y 27,23, y La 2,15ss. En David Cohen, François BRon y Antoine LonNet, Dictionnaire des racines sémitiques, ou attestées dans les langues sémitiques (Paris: Mouton-Leuven: Peeters, 1970-. 12 fasc.) fasc. 5, pág. 353, s. v. $h^{\prime} h$, en la entrada correspondiente, se dice de הֶָׁ que es un «cri de joie, cri pour exprimer le hennissement d'un cheval pendant le combat».

${ }^{75} \mathrm{La}$ riqueza del vocabulario relativo a la burla, el escarnio y el oprobio en la BH es

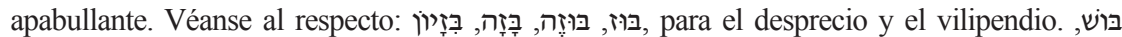

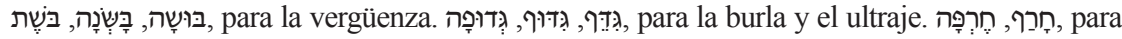




\subsection{La interjección הֶָָ.}

La risa suele considerarse como una vocalización no verbal, es decir, no significativa, aunque no habría que descartarlo. Cuando menos, pragmáticamente, e independientemente de la clase de palabras a la que se la adscriba y de cómo se la represente en la escritura, la risa tiene un potencial comunicativo indudable ${ }^{76}$.

Cabe preguntarse si, cuando alguien ríe, dice «ja-ja». Para R. R. Provine, no es así (vid. nota 27). En el texto bíblico hebreo, así parece: siempre הֶָָח (vid, infra). Provine, como se vio, considera la risa, filogenéticamente, una onomatopeya. La onomatopeya es un fenómeno fonético, no un tipo de palabra específico, consistente en la articulación de un sonido que sugiere un objeto o acción ${ }^{77}$ : un verbo, un nombre o una interjección pueden ser onomatopéyicos.

En la escritura, la risa se representa mediante la interjección «ja ja» o similar. Lázaro Carreter distingue tres clases de interjecciones: «apelativas», «exclamativas» y «representativas» («chist», «ay»y «pum», respectivamente), anotando que las últimas «son, a veces, verdaderas onomatopeyas» ${ }^{78}$, y especificando que las interjecciones pueden ser «propias» (como las señaladas en el paréntesis anterior, ejemplos de las tres clases según su función) o «impropias», es decir, palabras usadas

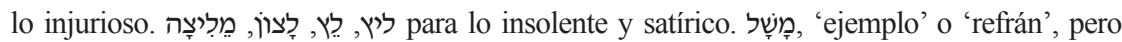
también, con sentido negativo, '(ser o hacer de alguien el) hazmerreír', como en Jb 17,6 o

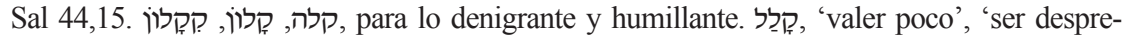
ciable'; en piel, 'ultrajar', 'insultar'; en hifil, 'despreciar'. קלס, 'ridiculizar', קקלק, 'irrisión,

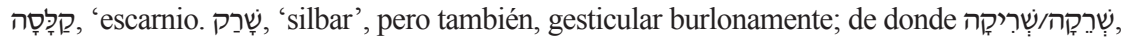

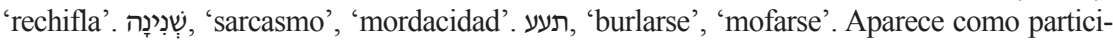
pio pilpel en Ge 27,12 y también como participio hitpalpel en 2Cr 36,16, junto con לעוּ y y '

76 Cf. Trouvain y Truong, «Laughter», pág. 341: «Sometimes laughter is considered to belong to the word class of interjections, i.e., particles with an affective component, often with a sound-imitative character. However, laughter as such, i.e., in its raw and untamed form, should be considered a non-verbal vocalization along with breathing sounds, sighing, or clicking»».

77 Fernando Lázaro CARreter, Diccionario de términos filológicos (Madrid: Gredos, $1977^{3}$ ) págs. 301-302, s. v. «onomatopeya».

${ }^{78}$ Una diferenciación entre interjección y onomatopeya puede verse en Maruszka MeINARD, «Distinguishing Onomatopoeias from Interjections», Journal of Pragmatics 76 (2015) págs. 150-168. 
eventualmente como interjecciones, sin serlo («idiablos!») ${ }^{79}$, con lo que parece que la interjección, más que un tipo de palabra, es también una función (funciones) expresiva ${ }^{80}$. Al ser la interjección una vocalización convencional que «encode[s] emotional states, mental attitudes and, in some models, communicative intentions», su clase o tipo léxico es heterogéneo y acoge «lexemes and constructions that differ considerably from a pragmatic, semantic, and structural perspective» ${ }^{81}$. Se podría aventurar que הֶאָ es una «interjección onomatopéyica propia».

El Dictionary of Classical Hebrew $(\mathrm{DCH})$ considera הָָ como interjección ${ }^{82}$, y también Targarona en su diccionario ${ }^{83}$, mientras que Schökel, en el suyo, no lo califica ${ }^{84}$. Tanto el $D C H$ como Schökel anotan todas sus apariciones en la BH (nueve; vid. infra), mientras que Targarona no ofrece ni una sola. Solo el primero ofrece una descripción del término: «interj. aha, as expression of satisfaction or joy, usu[ally] in response of another's misfortune» y señala que puede ir duplicado ${ }^{85}$. Ninguno hace referencia

79 LÁzaro CARreter, Diccionario de términos filológicos, pág. 243, s. v. «interjección».

80 Alexander ANDRAson, Irina Hornea y Marcus Joubert («The Structure of Interjections in Biblical Hebrew: Phonetics, Morphology, and Syntax», Journal of Hebrew Scriptures 20:1 [2020] págs. 1-43: 3) llaman a las interjecciones «propias» e «impropias», «primarias» $\mathrm{y}$ «secundarias», respectivamente, atendiendo a su grado de gramaticalización.

81 ANDRAson, Hornea y JouberT, «The Structure of Interjections», pág. 1. La discusión sobre la naturaleza y la clasificación de las interjecciones se remonta a los gramáticos griegos y aún sigue generando literatura, $c f$. la bibliografía en la referencia recién citada y en Felix AмEKA, «Interjections: The Universal yet Neglected Part of Speech», Journal of Pragmatics 18 (1992) págs. 101-118.

82 The Dictionary of Classical Hebrew $(D C H)$, ed. David J. Chines (Sheffield: Sheffield Academic Press, 1993-2016. Vols. I-IX) vol. II, pág. 484, s. v. הָָָח.

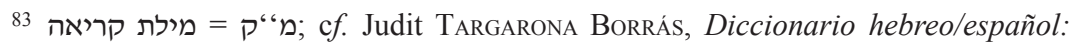
Bíblico, rabínico, medieval, moderno (Barcelona: Riopiedras, 1995) pág. 229, s. v. הֶ.

${ }^{84} \mathrm{C} f$. Luis Alonso Schoekel [sic], Diccionario bíblico hebreo-español (Valencia: Institución San Jerónimo, 1990. 2 vols.) vol. 1, pág. 168, s. v. הֶָָ.

${ }^{85}$ DCH vol. II, pág. 484, s. v. הֶָָ; sus negritas; cursivas añadidas. En A Hebrew and English Lexicon of the Old Testament, eds. Francis Brown, Samuel R. Driver y Charles A. BRIgGS (Oxford, 1906; reimpr. 1939) pág. 210, s. v. הָ, se dice que es una «interj. (onomatop.) expressing joy, Aha!». En Cohen, BRon y Lonnet, Dictionnaire des racines sémitiques, fasc. 5 , pág. 353, s. v. h'h he dice que הָָ es una interjección comparable a las de otras lenguas semíticas incluidas en la entrada $h-^{2} / h / w / y$ ( $c f$. págs. 352-353). En dicha entrada, se recogen los usos en hebreo, árabe, sudarábigo y etiópico, 
a la risa, y ni Targarona ni Schökel traducen como nada parecido a la interjección para la risa ( $\langle\mathrm{j} a » \mathrm{o}$ «je», simplificando), sino que sus traducciones son expresiones completas del tipo «ibien!», «ibravo!» (interjecciones impropias o secundarias) o interjecciones del tipo «iay!», en el caso de Targarona. El $D C H$, como significado y traducción ofrece aha, que en inglés corresponde al castellano «ajá», y que el Merrian-Webster define como interjección que expresa sorpresa, triunfo o escarnio (derision) ${ }^{86}$, pero no risa o irrisión, directamente. No muy diferente es la definición en el $D L E$ del correspondiente «ajá», aunque se incluye la 'aprobación' ${ }^{87}$.

הֶָָ aparece en nueve ocasiones en la $\mathrm{BH}$, ya sea aislada o reduplicada: Is 44,16; Ez 25,3, 26,2 y 36,2; Sal 35,21 y 25, 40,16 y 70,4, y Job 39,25 (no se tiene en cuenta aquí Sir 41,2). Měnaḥem ben Saruq, en su Maḥberet, incluye la expresión como tercera acepción en la entrada correspondiente a אח לאר (otra interjección, en una de sus acepciones) y dice de ella: תאמר לעת רבות משוש, anotando su aparición en Sal 35,21 y Ez 26,2, solamente. Rashi לשון consigna todas sus apariciones en Salmos, menos 35,25, y la denomina חדוה, «expresión de contento, alegría», en su comentario a Sal 70,4 ${ }^{89}$. De הָאָח en Job dice que es típico de momentos de felicidad, así como se dice cuando uno está a salvo ${ }^{90}$. Eliezer ben Yehudah, en su diccionario, la define como interjección (מילת קריאה) de alegría debida a la venganza, consignando su aparición en Is 44,16; Ez 25,3, 26,2 y 36,2; Sal 35,21 y 40,16, y Jb $39,25^{91}$. Como se observa, pese a las referencias a la alegría y el alborozo, ninguno las relaciona con el reír, ni siquiera con el reír revanchista.

entre otras lenguas, de expresiones como hĭ, hōy, hōhō, howa, hāhā, haw haw o haya. En general, tales expresiones indicarían sorpresa, dolor, placer, alegría, ironía o desdén.

${ }^{86}$ Merriam-Webster.com Dictionary, s. v. «aha», accesible en línea en <https:// www.merriam-webster.com/dictionary/aha> [último acceso: 25/04/2021].

$87 C f$. «ajá» en Real Academia Española, Diccionario de la lengua española, 23. ${ }^{a}$ ed. (versión 23.4 en línea, accesible en $<$ https://dle.rae.es> [último acceso: 25/04/2021]).

88 Sepher Mahberet Menahem, ed. Herschell FiLIPOwSKI (London-Edinburgh: Hevrat me'orere yeshenim, 1854) letra 48:2 א, Según la versión consultada en Sefaria.org, accesible en $<$ https://www.sefaria.org/Mahberet_Menachem?lang=he> .

${ }^{89}$ Cf. Rashi’s Commentary on Psalms, ed. Mayer I. GruBER (Leiden: Brill, 2004) pág. 836.

${ }_{90}$ Según edición del comentario de Rashi a Job accesible en $<\mathrm{https}: / / w w w . s e f a r i a$. org/Rashi_on_Job>. Rashi no comenta הֶָָח en Isaías. Para sus comentarios a en Ezequiel, vid. infra.

91 Ben Yehuda, Dictionary, vol. 2, pág. 1022, s. v. הָָ. 


\section{2. הֶָ como representación de la risa humillante}

En Ez 25,3.26,2 y 36,2 el profeta denuncia a Amón, Tiro y los enemigos de Israel, respectivamente, por sus ventajistas y hostiles risas ${ }^{92} \mathrm{y}$ como prólogo del anuncio de lo que les espera, del mal que les depara la acción divina. «Os reísteis del mal ajeno, de las desgracias de mi pueblo», viene a decir, «afrenta que os haré pagar». Que la risa expresada por la interjección es carcajada triunfal por ver al enemigo caer le queda claro a Rashi, que en comentario a Ez 36,2 dice que השון קול es . Los tres pasajes de Ezequiel nos dan una pista sobre cómo se verbalizaban las burlas tantas veces demonizadas en los Profetas, además de decirnos cómo se podía representar la particular vocalización de la risa en hebreo bíblico. En los tres casos se les afea a los enemigos de Israel y Judá su alegría por la caída y destrucción de estas, empleándose la misma fórmula: «Por cuanto... entonces, he aquí que Yo...».

Es de notar que ninguno de los tres oráculos dice: «Por cuanto os reísteis, diciendo...», sino: «Por cuanto dijisteis: JA JA [más la causa de la risa correspondiente]». La risa, por tanto, es expresada o representada siempre en estilo directo, al igual que en Salmos (vid. infra). Por eso la aparición de אמר en todos los casos: porque la carcajada forma parte de lo enunciado y es vocalización que antecede lo discursivo (vid. las observaciones de Provine y otros en apdos. 3 y 4).

El salmista, en los pasajes bajo escrutinio (y en tantos otros) pide la intervención divina contra sus enemigos, haciendo hincapié en las risas y las burlas de que es objeto. La risa es violencia que provoca y legitima más violencia (2Re 2,23-24) y por eso se pide expresamente la intervención de Yhwh. Sabe el salmista que peca quien zahiere y se alegra de los infortunios de quien sí cumple la Ley, y que no quedará impune (Pr $17,5)$.

Para los cuatro pasajes proféticos, Schökel, en la entrada de su diccionario (vid. nota 84), ofrece como traducciones expresiones del tipo

${ }^{92}$ En Isaías 44,16 parece que la «carcajada» la emite el profeta, pero la pone en boca del idólatra.

93 Según la edición de su comentario a Ezequiel accesible en $<\mathrm{https}: / / w w w . s e f a r i a$. org/Rashi_on_Ezekiel>. 
«¡vaya!» ${ }^{94}$, «¡magnífico!», «¡bien!»; para el resto no sugiere traducciones, sino que se limita a señalar que, en Salmos, expresa burla, y en Job, entusiasmo (vid. nota 73). Sin embargo, en la traducción que dirigió, Biblia del peregrino, se traduce siempre para Salmos, menos en 35,25, como «ja, ja» ${ }^{95}$ :

- 35,21: Se ríen de mí a carcajadas ${ }^{96}$ : Ja, ja, lo estamos viendo.

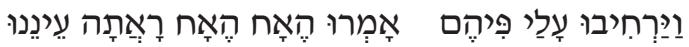

Rashi comenta de este versículo que הֶָָח הֶָר es expresión de quien se jacta de lo que ha deseado ${ }^{97}$.

- v.25: Que no canten victoria, que no piensen: ¡Qué bien! lo que queríamos

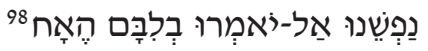

- 40,16: Queden mudos de vergüenza los que se burlan: Ja, ja.

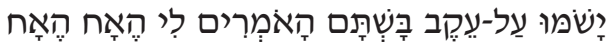

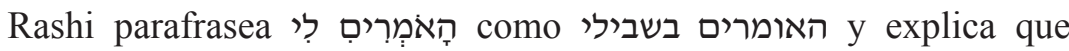
«cuando nos llega [a los judíos] una desgracia, se regodean en nuestra calamidad $\gg 99$.

- 70,4: Retírense derrotados los que se burlan: Ja, ja.

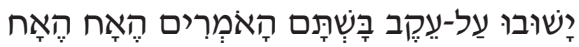

94 'Vaya' en castellano es también sustantivo: «burla o mofa que se hace de uno o chasco que se le da»; «vaya», en Real Academia Española: Diccionario de la Lengua Española, 23 $3^{\mathrm{a}}$ ed. (versión 23.4 en línea en https://dle.rae.es [acceso 25 de abril de 2021]).

95 Todas las traducciones de Alonso SchöKEl, Biblia, vol. 2, págs. 620, 631 y 673, respectivamente. En cursivas, la divergencia respecto al resto de rendiciones de הֶ. Misma traducción en Luis Alonso SchöKel y Cecilia Carniti, Salmos. Introducción, traducción y comentarios (Estella: Verbo Divino, 1992. 2 vols.). El salmo 70 sería bien el origen, bien una singularización de Sal 40,14-18 (Alonso ScHöKel, Biblia, vol. 2, pág. 631); $c f$. las notas textuales respectivas en $B H S$.

96 En castellano, «carcajada» es sustantivo onomatopéyico.

97 לשון מתהלל בתאות לבו, (Rashi’s Commentary, pág. 823).

98 La nota correspondiente en la BHS sugiere la reduplicación de la interjección. Rashi no comenta este verso, por lo que no se puede saber si sus fuentes incluían dicha reduplicación, como sí ocurre con la particula לִ en Sal 70,4 (vid. infra).

99 Rashi’s Commentary, pág. 825; la traducción es mía. 
La BHS sugiere la inclusión de ț tras el participio, como en 40,16. Rashi glosa la partícula, por lo que sus fuentes textuales la incluirían, y dice de ella que ha de entenderse como עָָ, «de mí, sobre mí» ${ }^{100}$. Sobre la interjección, además de observar su condición de expresión de alegría (vid. nota 89), añade que se da בראותו באויבו כרצונו, ex decir, cuando se ve a un enemigo (tan mal) como se desea ${ }^{101}$. ראה בר expresión de satisfacción por lo que le ocurre al otro, especialmente si es una desgracia (cf. Sal 37,34 y 54,9), con lo que queda claro que Rashi se refiere a que הָָ es una expresión de alegría de (en esta concreta ocasión) los enemigos por el mal acaecido al salmista.

Obsérvese que, para los casos en Salmos, en ningún caso Schökel traduce el verbo אמר, sino que identifica como enunciado completo todas las vocalizaciones, y el acto de habla, como burla, ya sea expresa $(40,16$ y 70,4), ya implícita $(35,21)$. En Sal 35,25 traduce el modismo אמר בלב, no

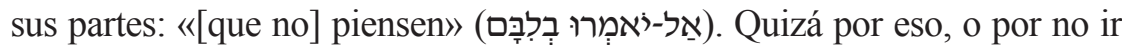
reduplicada, traduce la interjección como interjección impropia. Lo interesante es que puede que esté interpretando igualmente como modismo אמר y, por eso, para el resto de los casos jamás lo traduce «decir + interjección propia/impropia», como se suele hacer y como en su propio diccionario propone ${ }^{102}$. Parece que tendría claro que el significado idiomático no sería el literal en este caso (es decir: que el significado del todo no es el de la suma de las partes) ${ }^{103}$, y que la expresión אמר האח האח ni siquiera significaría «reír» (a carcajadas, si se abre mucho la boca: Sal 35,21), sino decir riendo, o reír + decir (burlona, mordaz o sarcásticamente).

La risa es ofensiva, hostil y degradante, sobre todo, por venir de quienes viene: de los «malos», de los que no cumplen con los estatutos,

100 Rashi's Commentary, pág. 836.

101 Rashi's Commentary, pág. 836.

102 Shlomo Weisblit («Psalm 70. A Personal Prayer» [en hebreo]. Beit Mikra: Journal for the Study of the Bible and Its World 20, fasc. 1974] א] págs. 76-78: 77) dice [1a traducción es mía]: «el modismo [לעג ובוז] הבטוי] señala burla y menosprecio como [en Sal 35,21]. También indica un grito de alegría por la desgracia, como [en Ezequiel 25,3]»; $c f$. además nota 7 en la misma página.

$103 C f$. Andy Warren-Rothlin, «Body Idioms and the Psalms», en Interpreting the Psalms: Issues and Approaches, eds. Phillip S. Johnston y David G. FirTh (Downers Grove, III: IVP Academic, 2006) págs. 195-212: 197. 
o peor, del idólatra. Porque cuando las tornas cambian, tras la intervención divina, y el injustamente tratado es ensalzado y prevalece, o el enemigo es juzgado y castigado, son los justos los que ríen y se alegran: Jb 22,19-20. Mientras tanto, el piadoso se queja de ser la irrisión de los demás y expresa el oprobio que le provocan las chanzas de sus enemigos (Sal 22,7-8; 44,14-16; 79,4; 123,4, etc.), y pide no burlarse del mal ajeno (Pr 24,17-18).

Es el ridículo lo que más duele y lo que más se teme, pero no solo en las «filas bíblicas», sino en todas las partes implicadas. Por eso el profetismo se recrea en las amenazas a las rebeldes Israel y Judá con ser objeto de burla y befa (Je 24,9-10; 25,15-18; 29,18; 44,12, etc.). Y por eso Ezequiel principia los oráculos reseñados «recordando» la hostil risa con que los enemigos celebraran las caídas de Israel y Judá: porque la revancha o el castigo será también una especie de «contra-risa» (Sal 2,4.59,9). Tomando el modelo de Martineau visto en el apdo. 4.1., resulta obvio que la risa hostil tiene unas consecuencias diferentes dependiendo de los sujetos implicados y de sus respectivas adscripciones sociales, así como de quién inicia la risa y a quién implica o involucra esta. Así, la risa puede consolidar un grupo, desmoralizarlo, desintegrarlo, fomentar las rencillas, etc. Y de la misma manera ocurriría con un individuo con respecto a sus correligionarios.

\section{Conclusiones}

Como se ha intentado mostrar, el estudio de la risa en la $\mathrm{BH}$ puede abordarse sorteando las cuestiones de lo risible como respuesta a lo cómico y al humor, y esquivando las disquisiciones acerca de las diferencias entre el humor «de allí y de entonces» y el «de aquí y ahora». Parece que una manera más provechosa de afrontar la supuesta relación causa-efecto entre los distintos fenómenos implicados es partir de las funciones que comparten, en tanto que signos o medios comunicativos. Así, en contextos sociales, se puede analizar cómo funciona la risa, cuando el escarnecimiento, la burla y el desdén se le unen, tanto en las interacciones dentro de un grupo como en las intergrupales. Algunos modelos creados por la sociología sobre el humor humillante o degradante pueden ayudar a entender mejor el papel de algunos tipos de risa, 
según se presentan y se los distingue en el texto bíblico. La condición de signos comunicativos compartida entre humor y risa permite la aplicación de estos modelos, basados en las funciones que ejercen ambos fenómenos, no en las causas o detonantes que los provocan, o en su supuesta relación uno-a-uno. Esto pasa por una caracterización de la risa no dependiente de sus causas, sino de su condición de vocalización estereotipada y universal de indudable valor comunicativo.

La expresión הֶָָח, en sus apariciones en la BH, se presenta en contextos intra-grupales e inter-grupales. Parece indudable su condición de interjección representativa propia, claramente onomatopéyica. Suele ir casi siempre reduplicada, lo que acentúa su especial naturaleza expresiva y estereotipada. Aparece como parte integrante de las enunciaciones y como vocalización que enmarca el inicio del contenido de lo verbalizado, y representa un tipo de risa hostil, burlona, degradante, que subestima a su objetivo. Aparece en contextos de rivalidad o competencia, ya en el seno de un grupo, ya en las relaciones entre dos grupos. Cotextualmente, se alude tanto a la función claramente hostil de la interjección הָָָ, como a las repercusiones que tiene la humillación que expresa, tanto en el/los sujeto/s riente/s como en su objetivo, sea este un individuo o un colectivo, internos o externos. Dichas repercusiones, en cualquier caso, pueden ser socialmente cohesivas / consolidadoras («lubricantes») o desmoralizantes / desintegrantes / conflictivas («abrasivas») para cada parte implicada.

Recibido: $11 / 01 / 2021$

Aceptado: 24/05/2021 
